\title{
SPL Risk Evaluation and Mitigation Strategies Terminology
}

National Cancer Institute

\section{Source}

National Cancer Institute. SPL Risk Evaluation and Mitigation Strategies Terminology.

NCI Thesaurus. Code C128490.

Risk Evaluation and Mitigation Strategies (REMS) are programs required by FDA and put in place by drug manufacturers to help ensure that the benefits of certain drugs outweigh their risks. The terminology is used as part of Structured Product Labeling to describe the requirements REMS Programs place on participants, including healthcare providers and patients, to help assure that the drug is used safely. 